\title{
Smart Multi-Aperture Radar Techniques for Spaceborne Remote Sensing
}

\author{
Marwan Younis, Federica Bordoni, Nicolas Gebert, and Gerhard Krieger \\ German Aerospace Center (DLR), Microwaves and Radar Institute \\ Email: marwan.younis@dlr.de
}

\begin{abstract}
The paper deals with the performance of next generation SAR sensors, here referred to as SMART (Smart Multi Aperture Radar Techniques) equipped with digital beamforming capabilities. Apart from representing a technological jump, these sensors offer the flexibility of actually deciding on the (possibly hybrid) mode(s) of operation on-ground after the data have been acquired. The paper presents the performance of SMART system configurations and modes of operation for a digital beamforming SAR which covers a large swath with a high resolution.
\end{abstract}

\section{INTRODUCTION}

If spaceborne synthetic aperture radar (SAR) sensors were categorized according to their operational flexibility, four generations could be identified. The first sensors were on/off sensors with a single fixed mode of operation (fixed beam, incidence angle, bandwidth, etc.) Later, sensors were developed which could be operated in multiple modes, such as StripMap, ScanSAR or SpotLight; however the macro-based commanding was basically restricted to selecting a specific mode without the flexibility to alter/access individual instrument settings. Current SAR sensors -third generation- offer a greater flexibility in commanding nearly each individual parameter of the instrument (see TERRASAR-X [1] for example). This can be understood as providing the basic building blocks to construct any operation mode in combination with any possible instrument setting. However, even current sensors do not offer a satisfactory solution to the "fundamental limitation of SAR sensors" which can be described in short as the incapability to simultaneously provide high resolution and wide coverage [2], instead they offer the flexibility to choose a compromise between high resolution or wide coverage.

Intensive research is on-going for a new generation of Smart Multi Aperture Radar Techniques (SMART) published for example in [3], [4], [5], [6]. The main innovative characteristic of this forthcoming generation of SAR systems is the use of multiple elevation and/or azimuth channels (see Fig. 1) combined with digital beamforming capability. This allows for the synthesis of multiple or dynamic digital receiver beams. In general SMART operational modes produce one or more digital receiver beams, each covering an unambiguous range segment on the ground. Utilizing the digital beamforming property of SMART sensors, these receiver beams are actually formed on the recorded echo signal without the necessity for analog beamforming capabilities. These beams can additionally be scanned in elevation direction to follow the echo on the ground (SCan-On-REceive), see [5], [7].
The paper presents the performance of a number of SMART systems utilizing various operational modes. We intentionally restrict the paper to the presentation of distinct performance results for each system. We refer to detailed literature for a description of the concepts behind these modes [3], [8], the multi-channel azimuth processing [4], [9], or possible demonstrator systems [7], [10].

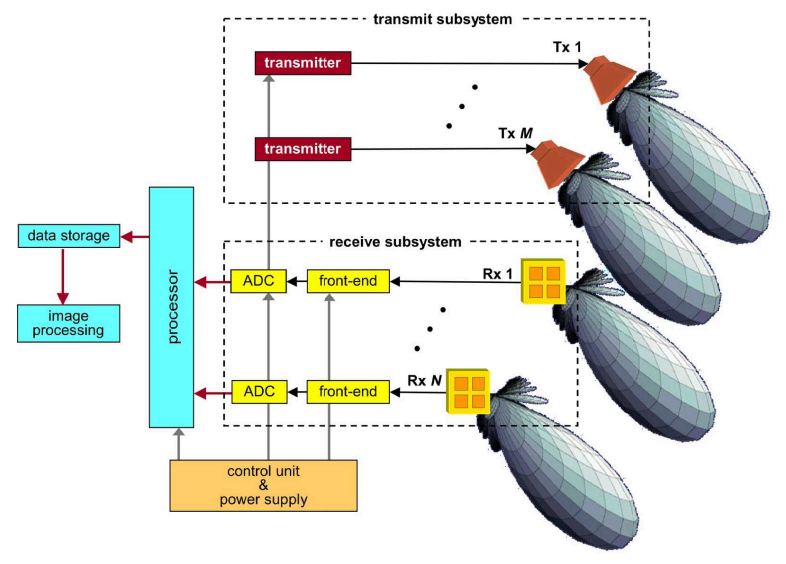

Fig. 1. Principle of Smart Multi Aperture Radar Techniques (SMART) systems.

\section{Performance Parameters}

In order to share a common basis for comparing the systems to each other we design all the systems so as to cover a swath width of $400 \mathrm{~km}$ with a $5 \mathrm{~m}$ azimuth resolution and a $80 \mathrm{MHz}$ chirp bandwidth for a $700 \mathrm{~km}$ orbit. Further, the average transmit power available to all systems was fixed to $1000 \mathrm{~W}$ while assuming a system noise temperature and feed loss of $420 \mathrm{~K}$ and $1 \mathrm{~dB}$, respectively ${ }^{1}$. For all systems considered here we assume that the elevation array (receive and/or transmit) allows for the suppression of nadir returns. This can be seen as a result of dealing with systems that have (digital) beamforming capabilities in elevation, basically enabling the nulling of the nadir echo. We note, that while this assumption influences the timing and thus the $P R F$ selection it does not contradict the principal operation of the systems and modes presented.

\footnotetext{
${ }^{1}$ We choose a constant system noise temperature and feed loss for simplicity and ease of comparison between the systems, well knowing that the actual values may vary for the different systems.
} 
The following performance parameters are investigated:

- range-ambiguity-to-signal ratio $(R A S R)$

- azimuth-ambiguity-to-signal ratio $(A A S R)$

- relative normalized power (scalloping)

- noise equivalent sigma zero (NESZ)

- scan-on-receive loss

Depending on the system being investigated only a subset of the performance parameters emphasizing the peculiarity of the system are presented in detail.

The description of the systems (referred to as system parameters) is mainly in terms of the transmit (Tx) and receive $(\mathrm{Rx})$ antenna size and the number of receive channels. In general, antenna heights are given with respect to the wavelength, while antenna lengths are given in absolute values, i.e. meters. Further, for each system, we specify the mode of operation through the number of bursts, where a StripMap multi-channel azimuth processing [9], [4] is used in the case of one burst and a ScanSAR multi-channel azimuth processing otherwise [11] (an exception is the multi-receive system in section VI where a conventional ScanSAR azimuth processing is used). The number of simultaneous SCORE beams in elevation direction is referred to as "fingers" and included in the system description. The system parameter table additionally includes the $P R F$ s, duty cycle, and number of sub-pulses.

\section{ClassicAl SCORE (HRWS) SySTEM}

Here the performance of a SCan-On-REceive system as presented in [12], [5] is investigated. However, in contrast to [5] the system is designed to achieve a $400 \mathrm{~km}$ swath with a $5 \mathrm{~m}$ resolution. The system parameters are shown in Table I.

\begin{tabular}{|lc|lc|}
\hline \hline Tx antenna height & $3 \lambda$ & & \\
Tx antenna length & $7.5 \mathrm{~m}$ & & \\
\hline Rx antenna height & $21 \lambda$ & elevation channels & 1 \\
Rx antenna length & $35.3 \mathrm{~m}$ & azimuth channels & 6 \\
\hline bursts & 1 (StripMap) & $P R F$ & $425 \mathrm{~Hz}$ \\
fingers & 1 & & $10 \%$ \\
sub-pulses & 1 & duty cycle & 10 \\
\hline \hline
\end{tabular}

CLASSIC SCORE (HRWS) SYSTEM PARAMETERS.

The noticeable long receive antenna is a consequence of the low PRF, which in turn results from the wide swath which is required to be free of blind ranges (transmit instances). Consequently multiple receive channels, i.e. digitalbeamforming in azimuth, is required to adequately sample the Doppler spectrum. This yields an azimuth resolution and $A A S R$ of $4.8 \mathrm{~m}$ and $-30 \mathrm{~dB}$, respectively, which do not show any significant variation over ground range.

The calculated RASR and NESZ are shown in Fig. 2 and represent an adequate performance over the swath. This is mainly because the $R A S R$ profits from the low $P R F$, while the long antenna results in a good NESZ value.

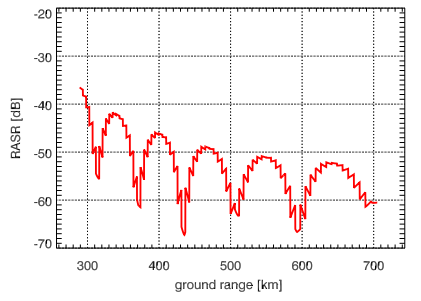

(a) range-ambiguity-to-signal ratio

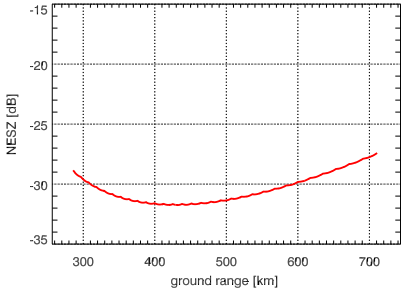

(b) noise-equivalent-sigma-zero
Fig. 2. Performance of classical SCORE (HRWS) system in terms of $R A S R$ and NESZ.

\section{SCANSAR SCORE SYSTEM}

The difficulties with the long receive antenna appearing for the system of section III may be overcome by choosing a burst operation mode, where multiple sub-swathes are successively illuminated, each with a different $P R F$. The system parameters for this mode of operation are shown in Table II where the $P R F$ selection is based on the timing diagram of Fig. 3 with a total of three sub-swathes and bursts. During each burst the transmit antenna array is steered to illuminate one sub-swath, while the receive array is used to form a sharp beam which tracks the pulse on the ground.

\begin{tabular}{|lc|lc|}
\hline \hline Tx antenna height & $6 \lambda$ & & \\
Tx antenna length & $1.8 \mathrm{~m}$ & & 1 \\
\hline Rx antenna height & $43 \lambda$ & elevation channels & 6 \\
Rx antenna length & $13.0 \mathrm{~m}$ & azimuth channels & 1215,1275, \\
\hline bursts & 3 (ScanSAR) & $P R F$ s & and $1155 \mathrm{~Hz}$ \\
fingers & 1 & & $10 \%$ \\
sub-pulses & 1 & duty cycle & $10 \%$ \\
\hline \hline
\end{tabular}

TABLE II

SCANSAR SCORE SYSTEM PARAMETERS.

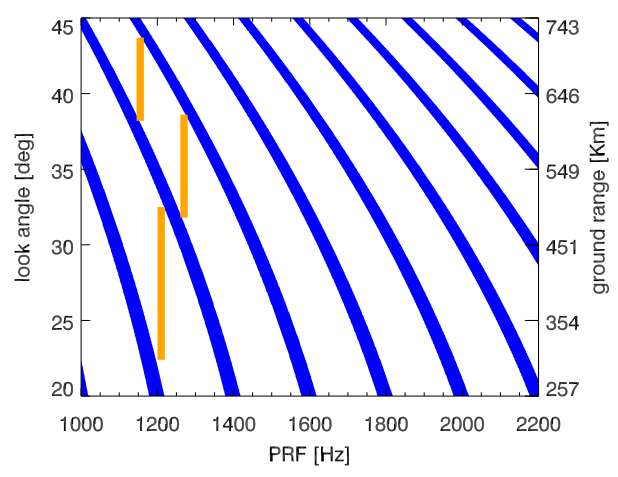

Fig. 3. Timing diagram of ScanSAR SCORE system.

The burst operation mode will result in an azimuth performance which depends on the along-track position of the scatterer relative to the beam center [11]. It is for this that the curves for the $A A S R$, and the scalloping in Fig. 4 are plotted versus the Doppler frequency, where $0 \mathrm{~Hz}$ represents the beam center. Each color in Fig. 4 represents one of the three sub-swathes. The azimuth resolution is nearly constant at $4.2 \mathrm{~m}$. 


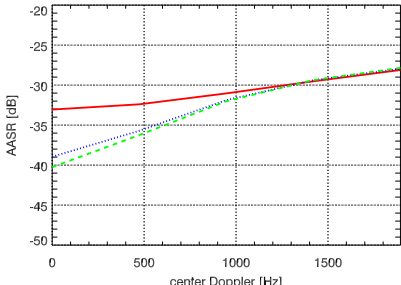

(a) $A A S R$

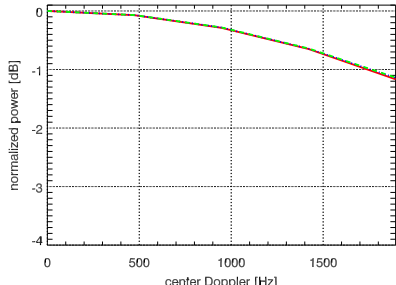

(b) scalloping
Fig. 4. Azimuth ambiguity and scalloping performance of ScanSAR SCORE system. The red, blue, and green curves correspond to sub-swath 1,2, and 3, respectively.

\section{Multi-Transmit SCORE System}

An extension to the ScanSAR operation mode is the use of sub-pulses as presented in [3], [8] and indicated in Fig. 5(a). During each transmission time, multiple sub-swathes are illuminated successively. To overcome the blind ranges between these sub-swathe a burst mode is added to close these gaps, as seen in the timing diagram of Fig. 5(b). On receive, multiple sharp beams (fingers) are formed, simultaneously collecting the signal from all sub-swathes of one burst.

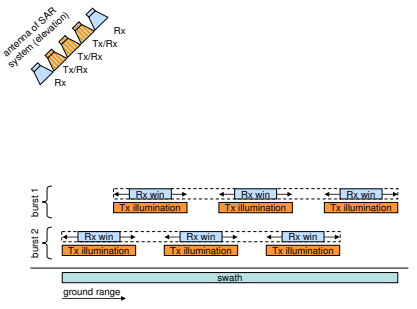

(a) operation mode

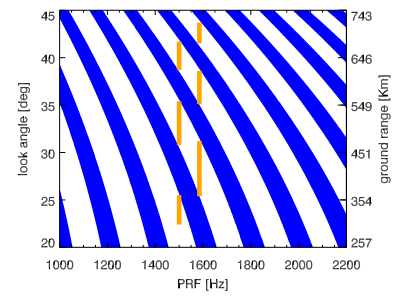

(b) timing diagram
Fig. 5. Multi-transmit operation mode and timing.

The system parameters for the multi-Tx system are given in Table III, where it is noted that the duty cycle is reduced with respect to the previous systems. The advantage of the sub-pulse operation is that higher $P R F$ values can be used which means shortening the receive antenna length. However, at the cost of a higher antenna required to adequately suppress the range ambiguities.

\begin{tabular}{|lc|lc|}
\hline \hline Tx antenna height & $16 \lambda$ & & \\
Tx antenna length & $2.8 \mathrm{~m}$ & & \\
\hline Rx antenna height & $97 \lambda$ & elevation channels & 3 \\
Rx antenna length & $10.8 \mathrm{~m}$ & azimuth channels & 6 \\
\hline bursts & 2 ScanSAR) & PRFs & 1500 \\
fingers & 3 & & and $1585 \mathrm{~Hz}$ \\
sub-pulses & 3 & duty cycle & $8 \%$ \\
\hline \hline
\end{tabular}

TABLE III

MULTI-TRANSMIT SCORE SYSTEM PARAMETERS.

The $R A S R$ is shown in Fig. 6 for all sub-swathes. The curve depends on the order of sub-pulse transmission, i.e. illumination, for example from near- to far-range or visa versa. However, the mean $R A S R$ value does not change considerably with the sub-pulse order. While sub-pulses allow for the more efficient use of transmit power they result in higher antenna for range ambiguity suppression.

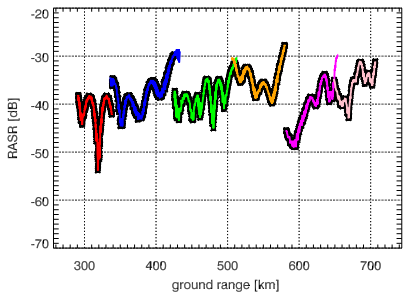

(a) far, mid, near sequence

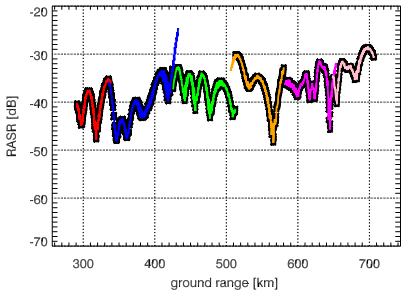

(b) near, far, mid sequence
Fig. 6. Range-ambiguity-to-signal ratio for the multi-transmit SCORE system for different sub-pulse sequences.

The high receive antenna will result in a reduced halfpower beamwidth compared, for example, to the systems in section IV. This, in turn, means a higher sensibility to topography changes within the swath. One figure-of-merit that reflects the system performance versus topography variation is the normalized SCORE gain, representing the variation of antenna gain for a fixed elevation. Here we assume the beam steering to be ideal in the case of $0 \mathrm{~m}$ terrain high ${ }^{2}$. A comparison of the SCORE gain for the ScanSAR and multitransmit SCORE systems is shown in Fig. 7 for a terrain height of $0 \mathrm{~m}$ and $1000 \mathrm{~m}$, respectively. While the SCORE loss is below $1.0 \mathrm{~dB}$ and would not be considered critical for either system, the higher loss in case of the multi-Tx system is recognized.

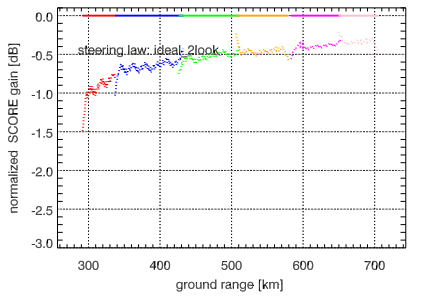

(a) multi-transmit SCORE system

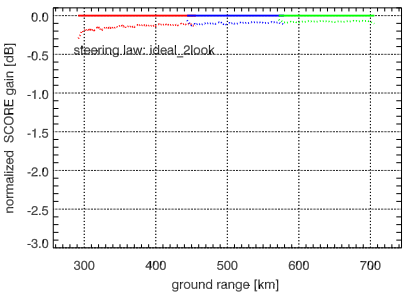

(b) ScanSAR SCORE system
Fig. 7. Comparison of normalized SCORE gain performance.

\section{Multi-ReCEIVE SCORE}

The sub-pulse operation mode can be avoided by illuminating the complete swath with a single small antenna, and using multiple sub-swathes on receive. Again burst mode operation is required to overcome the blind ranges. In such a multireceive SCORE system the timing is relaxed, since the total transmit time can be reduced, thus moving to higher $P R F$ values, as seen in Fig. 8.

In an extreme case the $P R F$ can be chosen high enough such that a single azimuth channel is sufficient to achieve the required resolution. The high $P R F$ comes at the expense of an increased number of simultaneous elevation beams as seen

\footnotetext{
${ }^{2}$ For a linear steering law and/or a single wide swath, even a terrain height of $0 \mathrm{~m}$ may already result in a non-negligible loss of gain.
} 


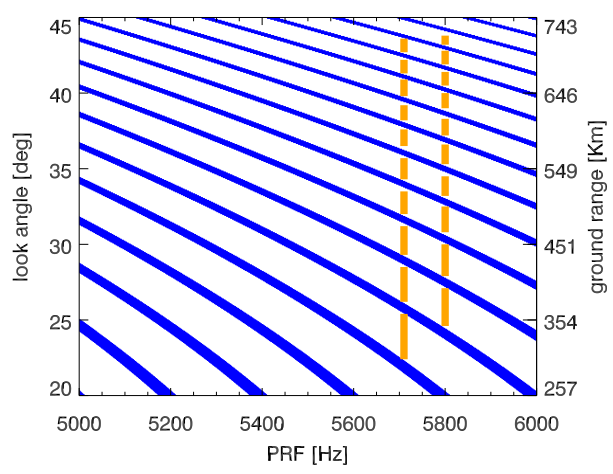

Fig. 8. Timing diagram of mult-receive SCORE system.

by the high number of fingers in Table IV. The short antenna length has to be compensated by a higher antenna in order to suppress range ambiguities.

\begin{tabular}{|lc|lc|}
\hline \hline Tx antenna height & $3 \lambda$ & & \\
Tx antenna length & $3.5 \mathrm{~m}$ & & \\
\hline Rx antenna height & $158 \lambda$ & elevation channels & 10 \\
Rx antenna length & $3.5 \mathrm{~m}$ & azimuth channels & 1 \\
\hline bursts & 2 (ScanSAR) & PRFs & 5710 \\
fingers & 10 & & and $5800 \mathrm{~Hz}$ \\
sub-pulses & 1 & duty cycle & $10 \%$ \\
\hline \hline
\end{tabular}

TABLE IV

MULTI-RX SCORE SYSTEM PARAMETERS.

A single azimuth channel simplifies the processing (classical StripMap) since it overcomes the problem of non-uniform sampling associated with digital beamforming in azimuth. However, the system now also lacks the flexibility of multiple azimuth channels. As shown in Fig. 9 this results in a degraded azimuth performance with respect to the previous systems (note that the large number of curves in the figure result from a total of 20 sub-swathes). The same is true for the $A A S R$ which is approximately constant at $-25 \mathrm{~dB}$.

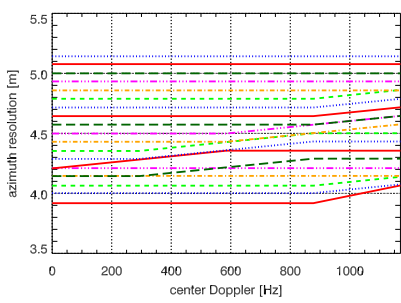

(a) azimuth resolution (b) scalloping

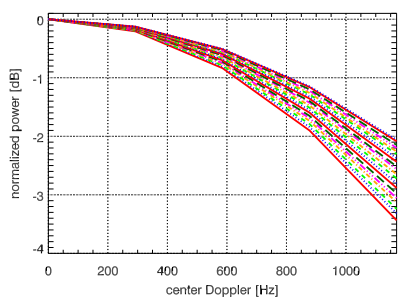

Fig. 9. Azimuth performance of multi-Rx SCORE system in terms of azimuth resolution and scalloping.

The $R A S R$ performance shown in Fig. 10(a) results from a receive antenna sufficiently heigh such that the nearest range ambiguity is positioned outside the main lobe. The NESZ in Fig. 10(b) reflects the broad pattern of the transmit antenna in conjunction with the high gain of the receive beams.

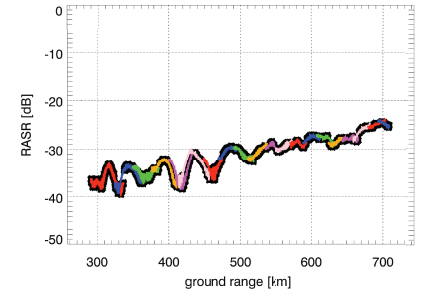

(a) range-ambiguity-to-signal ratio

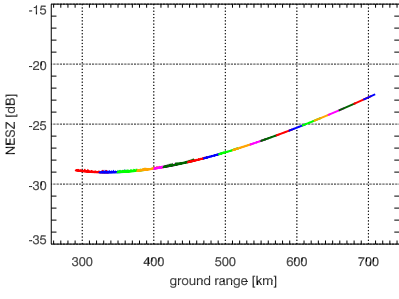

(b) noise-equivalent-sigma-zero
Fig. 10. Performance of multi-receive system in terms of RASR and NESZ.

\section{CONCLUSION}

The paper presents the performance of various SMART systems, all designed to cover a $400 \mathrm{~km}$ swath with a $5 \mathrm{~m}$ azimuth resolution, which is way beyond the performance of current SAR systems. It was shown, that in principle the design goal can be achieved. However, the investigated systems and modes show advantages and disadvantages in terms of required antenna size and channels, operation complexity, and power. Any final system conception requires a careful trade-off analysis between achievable performance and system parameters.

\section{REFERENCES}

[1] TerraSAR-X website. German Aerospace Center. [Online]. Available: http://www.dlr.de/tsx

[2] A. Freeman, W. Johnson, B. Huneycutt, R. Jordan, S. Hensley, P. Siqueira, and J.Curlander, "The 'Myth' of the minimum SAR antenna area constraint," IEEE Transactions on Geoscience and Remote Sensing, vol. 38, no. 1, pp. 320-324, Jan. 2000.

[3] G. Krieger, N. Gebert, and A. Moreira, "Multidimensional waveform encoding for synthetic aperture radar remote sensing," IEEE Transactions on Geoscience and Remote Sensing, vol. 46, no. 1, pp. 31-46, Jan. 2008.

[4] N. Gebert, G. Krieger, and A. Moreira, "Digital beamforming on receive: Techniques and optimization strategies for high-resolution wideswath SAR imaging," IEEE Transactions on Aerospace and Electronic Systems, 2008, accepted for publication.

[5] M. Süß, M. Zubler, and R. Zahn, "Performance investigation on the high resolution, wide swath SAR system," in Proc. European Conference on Synthetic Aperture Radar EUSAR'2002, Koeln, Germany, June 2002, pp. 187-190.

[6] M. Younis, C. Fischer, and W. Wiesbeck, "Digital beamforming in SAR systems," IEEE Transactions on Geoscience and Remote Sensing, vol. 41, no. 7, pp. 1735-1739, July 2003.

[7] C. Fischer, C. Schaefer, and C. Heer, "Technology development for the HRWS (High Resolution Wide Swath) SAR," in International Radar Symposium IRS'07, Sept. 2007.

[8] G. Krieger, N. Gebert, M. Younis, F. Bordoni, A. Patyuchenko, and A. Moreira, "Advanced concepts for ultra-wide swath SAR imaging," in Proc. European Conference on Synthetic Aperture Radar EUSAR'08, Friedrichshafen, Germany, June 2008.

[9] G. Krieger, N. Gebert, and A. Moreira, "Unambiguous SAR signal reconstruction from non-uniform displaced phase centre sampling," IEEE Geoscience and Remote Sensing Letters, vol. 1, no. 4, pp. 260264, Oct. 2004.

[10] J. Kim, M. Younis, D. Becker, and W. Wiesbeck, "Experimental performance analysis of digital beam forming on synthetic aperture radar," in Proc. European Conference on Synthetic Aperture Radar EUSAR'08, Friedrichshafen, Germany, June 2008.

[11] N. Gebert, G. Krieger, and A. Moreira, "Multi-channel ScanSAR for high-resolution ultra-wide-swath imaging," in Proc. European Conference on Synthetic Aperture Radar EUSAR'08, Friedrichshafen, Germany, June 2008.

[12] M. Süß, B. Grafmüller, and R. Zahn, "A novel high resolution, wide swath SAR," in Proc. Int. Geoscience and Remote Sensing Symposium IGARSS'01, vol. 3, 2001, pp. 1013-1015. 